\title{
Studies Measuring the Rotation Angle of the Driver's Face Method Based on Three-Dimensional Image Measurement
}

\author{
Li Dongmei $^{1, *}$ and Zhang Shengmin ${ }^{2}$ \\ ${ }^{I}$ School of Computer \& Information Engineering, Nanyang Institute of Technology, Henan, Nanyang, 473000, P.R. Chi- \\ $n a ;{ }^{2}$ Institute of Information Engineering, Kaifeng University, Henan, Kaifeng, 475004, P.R. China
}

\begin{abstract}
Among existing head orientation methods, two-dimensional image measurement method has been widely used. However, this method is greatly influenced by ambient light, and the angle of rotation could be too wide to measure. To avoid this, this study uses three-dimensional image measurement technology which is based on stripy light to collect three-dimensional data of human face, and head orientation can be detected by analysis. The author takes advantage of the human nose as a feature point, and puts forward a method to detect the peak point of the nose from the wing. Finally, the head orientation can be detected through the nose information. In this experiment, the head model is used as the measurement object, and the experimental device based on visible light projection is constructed. The experimental results show that under the condition of infrared light projection, the method adopted in this paper can meet the measurement requirement in the range of \pm 50 degrees.
\end{abstract}

Keywords: 3D image measurement, head orientation, nose, image processing.

\section{INTRODUCTION}

In modern society, people's living standards have been greatly improved, and car ownership has increased rapidly. Therefore, people pay more and more attention to the safe driving, and the research on the safe driving technology is more and more thorough. It is fully estimated that nearly half of all traffic accidents in the world are caused by fatigue driving. According to the statistics of the China Ministry of Communications, $40 \%$ of the car accidents in China are caused by this factor. Only in 2008, the road traffic accidents in China have occurred to 265204 events, resulting in 73,484 people killed, and 304919 people injured, with direct economic losses of 1.01 billion yuan. Among them, more than $60 \%$ of the traffic accidents are due to the distractible driver's fatigue [1]. As a result, attention is being given to the driver's attention. As we all know, the normal driving process of the driver's face should be facing the front. In the process of driving, if the driver's face deviates from the front of a larger angle, then it is prone to traffic accidents. Therefore, the study of the driver's face orientation is of great significance to the monitoring of the driver's attention.

For the detection of careless driving behavior, it is necessary to detect the direction of the driver's line of sight, and the direction of sight is determined by the direction of rotation of the face and the direction of the eye. Such as the driver's face and eyes are facing the front, comparing the cases that the driver's face turns to left 30 degrees but his eyes turn to right 30 degrees, they are all viewing front, but the area of sighting is obviously different, which is defined as a different line of view. In the paper, the direction of rotation of

*Address correspondence to this author at the School of Computer and Information Engineering, Nanyang Institute of Technology, Nanyang, 473004,P.R. China; Tel: 13803770071; E-mail: ywind2005@163.com of the face is studied, and a method for detecting the direction of rotation of the driver's face is presented.

\section{METHOD FOR MEASURING ROTATION ANGLE OF FACE}

\subsection{Measurement of Face Rotation Angle Based on Two -} dimensional Image Measurement Method

A face orientation analysis method based on the skin color characteristics and ratio of the left and right face skin area was proposed by Li Yang and others in Zhejiang University [2]. We define it as unsecured when the face rotation angle is greater than $15^{\circ}[3,4]$, which can be divided into three parts: imaging method, facial feature extraction and orientation analysis.

\section{(1) Imaging Method}

Because of the light conditions in everyday pictures taken when extracting the face or eye area, which is strongly affected by the brightness of the light. It is difficult to get a clear stable image in the very weak light at night or in the rather strong light under the direct sunlight injection. In this paper, the method of near infrared ray projection is proposed, which can avoid the influence of environmental light on the subsequent image processing, and does not cause any burden to the driver.

\section{(2) Facial Feature Extraction}

The author extracts the face and eye regions by means of the error back propagation (EBP) neural network, and judges the center of the face area and the center of the eye area. The location of the two nostrils was determined by the separability filter method, and the center of the nose was defined as the central position of the nose. 


\section{(3) Orientation Analysis}

First of all, the distance between the left and right eyes of the driver is calculated in advance, which is regarded as the known quantity, as shown in Fig. (1). In the experiment, the distance between the two eyes of the same driver is $\mathrm{D}^{\prime}$, and the distance between the nostrils and the eyes is $D_{1}$ and $D_{r}$, as shown in Fig. (2). We can get the face rotation angle $\theta$ in Fig. (2) according to the following formula (1). When $D_{1}$ is greater than $D_{r}$, the drive's face has turned to right, and turned to left in vice versa.

$\theta=\cos ^{-1}\left(\frac{D^{\prime}}{D}\right)$

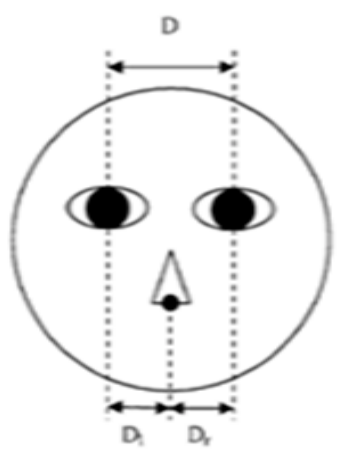

Fig. (1). Toward the front.

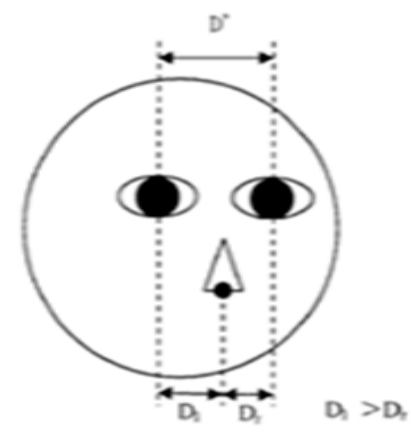

Fig. (2). Right deflection angle $\theta$.

The results show that the average error is $5.4^{\circ}$, the maximum error is $11.3^{\circ}$, the measurement range is $-60^{\circ}$ to $40^{\circ}$. The error increases with the increase of rotation angle.

The main advantage of the method: Firstly, the use of near-infrared light projection method will not give any burden for the driver and can avoid the impact of ambient light on the subsequent image processing effectively. Secondly, the measurement range is $-60^{\circ}$ to $40^{\circ}$, which is larger than the first method. Disadvantages: the method of extracting facial features based on BP neural network has a large amount of calculation and the experimental results are not accurate.

\subsection{Measuring the Rotation Angle of the Face Based on the Three-Dimensional Approach}

"An FPGA Implementation of Face Detection Recognition System for automobile using Impulse $\mathrm{C}^{\text {" introduces the }}$ method of measuring the Angle of the Face [5]. Due to the prior definition, when the driver's face rotation angle is greater than 15 degrees after unsafe driving, the target measured in this method is defined as 15 degrees. In order to avoid the impact of ambient light, the method also proposed a method of projection with infrared light. The measurement of the rotation angle of the face is also based on the detection of feature points, so it contains two parts: the threedimensional data measurements and the rotation angle measurements.

\subsubsection{Three-Dimensional Data Obtained}

The algorithm of face detection based on twodimensional image is more complex, and it is difficult to detect. In order to simplify the algorithm and reduce the computational cost, the company proposes a method of continuous projection with stripes [6]. The flow of the method is as follows: First, a horizontal stripe of infrared light is used to scan the driver's face. In the meanwhile, pictures of the driver are continuously photographed by an infrared camera from different angles. And then the three-dimensional coordinates for the driver's face can be achieved by using the method of three-dimensional image measurement.

\subsubsection{Rotation Angle Measurement}

The person's head as a sphere, from top to bottom is a round, the center position $\left(\mathrm{x}_{0}, \mathrm{y}_{0}, \mathrm{z}_{0}\right)$ of the sphere can be obtained by using the least square method of the three dimensional coordinate information. Then we calculate the $3 \mathrm{D}$ data of the face to the center of the distance, the furthest point from the center of the circle is defined as the nose feature point, and its coordinates are $\left(\mathrm{x}_{\mathrm{n}}, \mathrm{y}_{\mathrm{n}}, \mathrm{z}_{\mathrm{n}}\right)$, and the rotation angle of the face can be calculated by changing the polar coordinates [7].

\subsubsection{Experimental Results}

This method is still applicable when the driver wears glasses. "A study on the facial angle detection using the 3-D measurement technique" uses a three-dimensional measurement of fringe light projection method and feature point detection method based on the nearest point of the nose, and gives the experimental results. According to the experimental results, the maximum error is 1.1 degrees in the positive and negative rotation direction within 20 degrees, and the average error is 0.73 degrees.

\subsubsection{Experimental Methods}

The advantages of this method: The infrared projection method avoids the effects of ambient light; Wearing glasses and masks under the same conditions can be detected; Based on the three-dimensional image detection method, the accuracy of the measurement results is high in the range of about 20 degrees rotation. Disadvantages: due to the limitations of the nose point detection method, the measurement range can only be in the range of plus or minus 20 degrees. It is necessary to improve the measurement range of the driver's face rotation angle as auxiliary information for detecting the driver's proper driving. 


\section{FACE ROTATION ANGLE MEASUREMENT BASED ON VISIBLE LIGHT PROJECTION}

\subsection{Detection of Nose Vertex}

When the driver's face rotation angle is away from the forward direction for more than 90 degrees, the driver can't watch the front clearly even if he tries to do that with his eyes as far as possible. This condition is defined as non-safe driving. In this paper, the measurement range for the target is confined within a rotation range of 90 degrees in both directions [8]. By observing the three-dimensional data in the range of 90 degrees of rotation, we can conclude that the nose is always visible in this range. But when the rotation angle is large, only one side of the nose can be seen, and the location of the nose vertex can be observed. Thus we take the nose as a feature point of detection.

Each person's nose has two nose ends, the place where each nose end is connected to the face is a recess, and the center of the two recesses is nose vertex. Here we will call these two recesses as the two $\mathrm{H}$ slots of the nose. When the face is rotated, we can at least observe a groove. Therefore, this paper presents a method to determine the characteristic points of the nose by detecting grooves.

Since the three-dimensional measurement based on the projection light can't detect the shape information of the masked portion, the number of grooves that can be detected is different depending on the rotation angle of the face. When the rotation angle is small or close to the front, the two grooves can be detected, and if the rotation angle is large, only one can be detected. The concrete method is shown in Fig. (3).

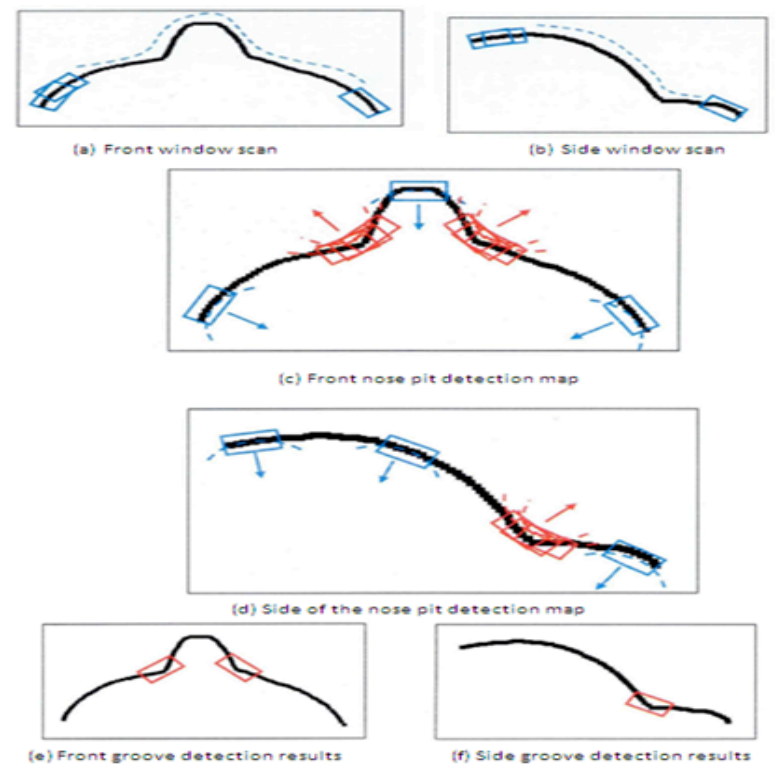

Fig. (3). Nose pit detection schematics.

Fig. (3a) is the three-dimensional data image of the face in the case of the front view. Fig. (3b) is a three-dimensional data image of a human face with a large rotation angle. In any case, the groove detection method is the same. Firstly, the point group in the three-dimensional data image is segmented from left to right with a certain step size and window.
In this paper, 40 data points are set as a window, with 10 points for the step, the blue frame in the Fig. (3a) and Fig. (3b) is a window, such as the blue dotted line by setting the same step size for scanning from left to right. And then the data points of each window are rounded to calculate the position and radius of the center of the circle. As shown in Fig. (3c) and Fig. (3d), when the center of the circle is pointed to the face contour, the window is defined as Non-groove, as shown in the blue window. On the other hand, when the center of the circle points to the outside of the curve, the figure is represented by a red window, the window is defined as a suspected groove area, where the arrow points to the center of the circle [9]. According to the different settings of the window and the step size, multiple consecutive windows may be suspected as groove area, the minimum radius of a plurality of continuous grooves in the window is set as a nose groove. Finally, the detection results of the groove are shown in Fig. (3e) and Fig. (3f). If two or more nose grooves are detected, the window and step are reset, and the nose Hgroove area is detected again until one or two nose grooves are detected.

\subsection{Experimental Measurements of the Projection Sys- tem Based on a Visible Light Face Rotation Angle}

In order to reduce the influence of ambient light, the experiment was carried out under the condition of no illumination and sunlight. In this experiment, the head model was used as the experimental object, and the experiment was carried out in the environment of Fig. (4).

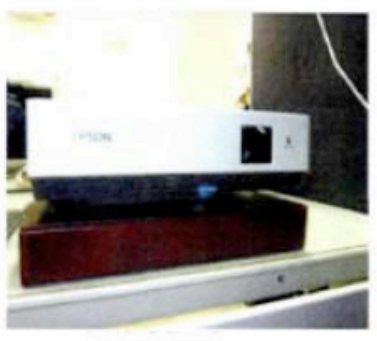

(a) Experiments proector

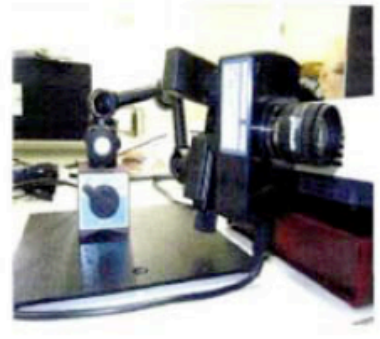

(c) Experiments camera

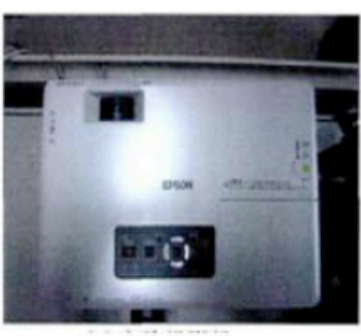

(b) Experiments proector

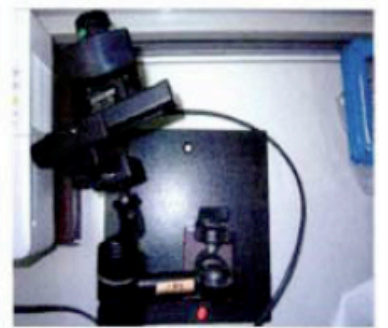

(d) Experiments canera
Fig. (4). Experimental device.

Fig. (5) is the experimental data graph, the face rotation angle of the experiment is measured to turn left 90 degrees to the right 90 degrees, measuring every 10 degrees in this range. The image on the left is captured by the camera, and the image on the right is a three-dimensional image. The yellow part is the detected nose groove, the red dot is the detected nose point. Fig. (5a) is the experimental image in 
the front view. Fig. (5e) is the projected image of the corresponding three-dimensional data in the $\mathrm{X}-\mathrm{Z}$ plane. Two grooves in Fig. (5a) were observed on the face. Correspondingly, the yellow region in Fig. (5e) is the detected two grooves, and the middle of the red dot is the detected nose point; Fig. (5b) is an experimental image that is rotated to $50^{\circ}$, Fig. (5f) is the projected image of the corresponding three-dimensional data in the $\mathrm{X}-\mathrm{Z}$ plane. As shown in the figure, this angle can also detect the two grooves of the nose, the nose is detected at the middle of the groove; Fig. (5c) is an experimental image that turns right at $60^{\circ}$, and Fig. (5g) is the projected image of the corresponding three-dimensional data in the $\mathrm{X}-\mathrm{Z}$ plane. As shown in the figure, we will find that there are some sparse points in the middle of the blue area. This reason is that no light was irradiated on the right half of the nose in this case, and no data are derived correspondingly. The resulting image should be the abscissa $X$ continuous, but the vertical axis $\mathrm{Z}$ will be intermittent. Fortunately, before we deal with the data, we're going to smooth the data. The value of each point is taken by the average of the two points next to it, so you have a picture of a sparse cluster at the discontinuities. Two grooves of the nose are also detected in Fig. (5g) after the smooth even if the nose $\mathrm{H}$-groove on the right side is actually not seen in Fig. (5c). Fig. (5d) is an experimental image that turns right at $70^{\circ}$, and Fig. (5h) is the projection image of the corresponding threedimensional data in the $\mathrm{X}-\mathrm{Z}$ plane. The projected image at $50^{\circ}$ is similar to that of $40^{\circ}$, except that the angle is different and both nose grooves and the nose points are detected in the middle.

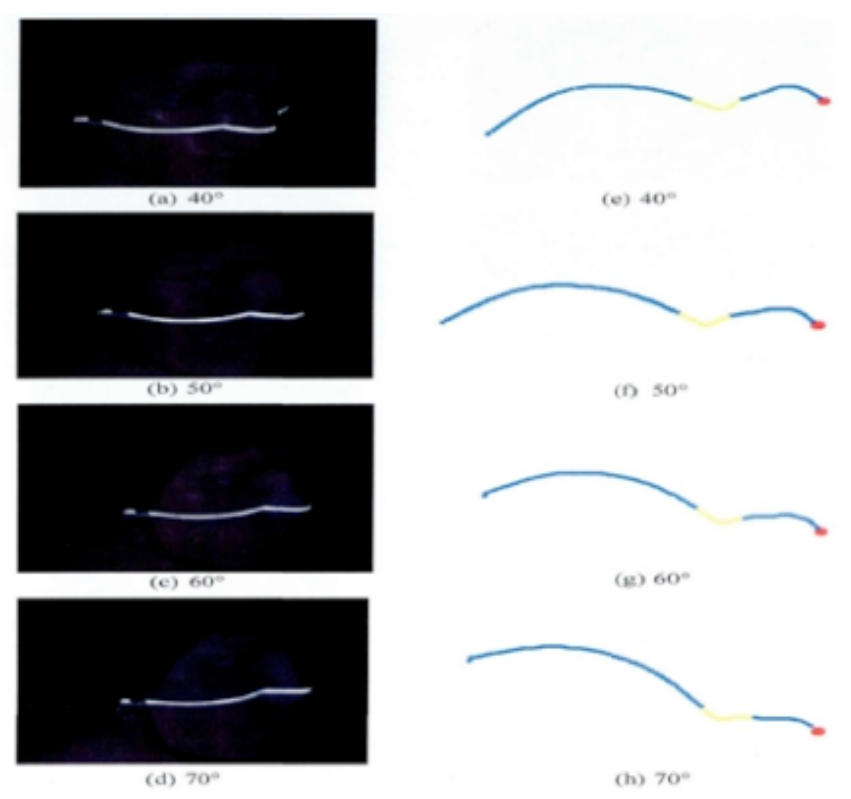

Fig. (5). Experiments image.

\subsection{Experimental Error Analysis}

By observing the three-dimensional data plotting the stripe, we can roughly deduce the experimental error in two aspects:(1) the selection of the nose feature points;(2)when calculating the angle of rotation, the error calculation method of reference image and measurement image integration.

\subsubsection{Nose Feature Point Selection}

Analysis of the three-dimensional data on the right side of the observation graph can find that the selection of the vertex of the nose is not accurate when the rotation angle is in the range of $40^{\circ}$ to $60^{\circ}$. When there is only one of the nose grooves, the nearest edge is judged as the top of the nose, so the detected nose point is the position of the red dot in the figure [10]. In the rotation angles between $40^{\circ}$ and $60^{\circ}$, more than half of the nose part can be deduced from the scanned images, and the edge point in the groove is actually not the real nose tip. When the angle is greater than 60 degrees, due to the large angle of rotation, the nose part is just half, and the edge point in the groove is just the real nose vertex. The position determination error for choosing the nose tip is smaller relative to that at the rotation angles between $40^{\circ}$ and $60^{\circ}$. The error reaches to maximum at the rotation angles between $40^{\circ}$ and $60^{\circ}$ due to upper mentioned reason. This should be regarded as a disadvantage for the selection of nose feature point.

\subsubsection{Integrated Error Calculation Method}

In the experiment, the light that was closer to the projectors in the object was stronger, and the three-dimensional data obtained was more intense. Therefore, the density of the obtained three-dimensional data points is not the same, which will affect the calculation of the integration error. In this paper, we use the coincident nose vertex as the reference point, and the datum points of the reference image as the datum. When there are data points around the nose in the measured image, the $\mathrm{X}$ coordinate in the reference image for the first data point $\mathrm{N} 1$ on the right side of the nose vertex is recorded in advance. Then from the first data point on the right side of the nose vertex in the measured image, stepped scanning is taken from left to right, and the nose point is recalibrated. We calculate the closest point between $\mathrm{Ml}$ and N1 of the X coordinate. This procedure should be done repeatedly until either of the data in the reference image or the measurement image ends. The data on both sides of the nose should be processed in this way, and a group of L1 values are derived. Finally the L1 values are superimposed as one value.

\section{MEASURING THE ROTATION ANGLE OF THE FACE BASED ON INFRARED LIGHT PROJECTION}

\subsection{System Components}

To reduce the burden on the driver and to eliminate the impact of ambient background light, infrared light is chosen as the projection light. In order to catch the infrared light by camera which is invisible to human eyes, a special filter is added in front of the camera (hereinafter referred to as infrared camera). The visible light will be filtered and only the infrared light could be recorded by the camera. The system's composition is basically the same as the visible projection system, but instead of replacing the projector with

a red light emitter, the normal camera is replaced with an infrared camera.

Because the infrared camera can shield the visible light, the experiment was carried out under the condition of ordinary illumination. The experimental setup is sketched in Fig. 
(6). In Fig. (6a), the near-infrared light emitting device Luminar Ace LA-100IR is used. Its emitted light wavelength is about $840 \mathrm{~nm}$ which is near the infrared wavelength. The near-infrared light in this waveband is harmful to the human eyes. But at this stage, the object is the head model, and the actual application will be applied to the infrared projection that does not harm the human body. In Fig. (6b), an infrared light emitter is used, and in Fig. (6c) and Fig. (6d), both kinds of low sensitivity industrial infrared cameras specific for the virtual head model are used. In the future, a high sensitivity infrared camera will be the first choice for the actual application.

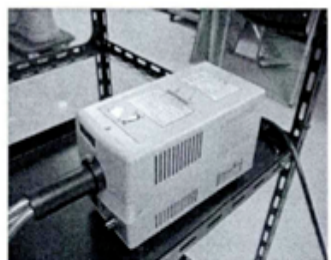

(a) Infrared light emitting means

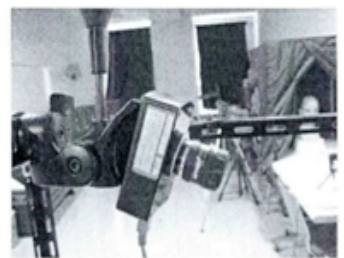

(c) Infrared Camera

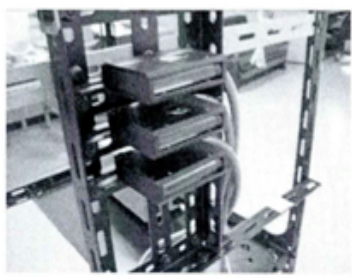

(b) Infrared light projection means

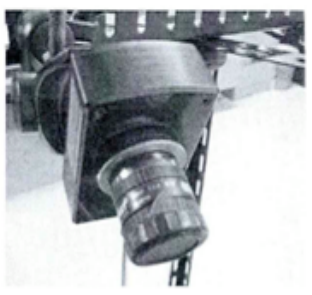

(d) Infrared Camera
Fig. (6). Experimental device.

\subsection{Experimental Results}

The experimental data images are shown in Fig. (7). The measurement is taken for the rotation angle of the face from $-90^{\circ}$ to $+90^{\circ}$ (in this paper, the left turn is marked with "-", and the right turn is marked with "+"). Every ten degrees is set as one step. The image on the left is captured by the camera, and the image on the right is a three-dimensional image. The yellow part is the detected nose groove, and the red dot denotes the detected nose apex. Compared to the image under visible light projection, the stripes are not located at the highest point of the nose apex, but slightly upside. The contour of the stripes is more moderate than that under visible light.

Fig. (7a) is the experimental image from the front view. Fig. (7e) is the projected image of the corresponding threedimensional data in the $\mathrm{X}-\mathrm{Z}$ plane. In Fig. (7a) two grooves are seen on the face, and in Fig. (7e) the detected two grooves are marked in yellow part, with a red dot in the middle of this part denoting the nose apex. The nose apex is figured out correctly; Fig. (7b) is the experimental image at a rotated angle of $+10^{\circ}$. Fig. (7f) is the projected image of the corresponding three-dimensional data in the $\mathrm{X}-\mathrm{Z}$ plane. The stripes are continuous at this angle, and two grooves of the nose are detected. The nose apex is also figured out correctly in the middle of the grooves. Fig. (7c) is the experimental image at a rotated angle of $+20^{\circ}$, and Fig. $(7 \mathrm{~g})$ is the projected image of the corresponding three-dimensional data in the $\mathrm{X}-\mathrm{Z}$ plane. In Fig. (7c), more light is projected on the right half part of the face, but the light at the nose apex point is a little intermittent more or less. This part of the projection area must be greater than we set before. In the Fig. (7f), we can't draw the fringe on the right side of the face, this is because there is only half of the face when it comes to judging and integrating the nose. The projection stripes under infrared light are not as complete as those under the visible light, so we can trickily discard the disconnected stripe points and just take the most intense part for further treatment. This can reduce the amount of calculations as well as the errors introduced from the incomplete stripes. The image in Fig. (7g) is thus derived, and one groove and the nose apex are correctly detected out. Fig. (7d) is an experimental image that turns right at $30^{\circ}$, and Fig. (7h) is the projection image of the corresponding three-dimensional data in the $\mathrm{X}-\mathrm{Z}$ plane. The projected image at $30^{\circ}$ is similar to that of $20^{\circ}$, except that the angle is different and both nose grooves and the nose points are detected in the middle.

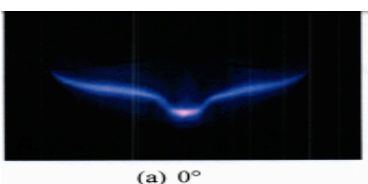

(a) $\mathrm{O}^{\circ}$

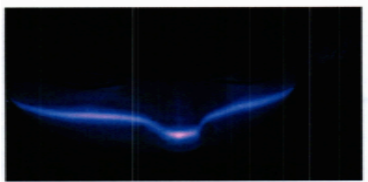

(b) $10^{\circ}$

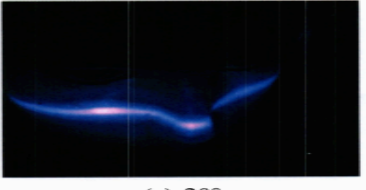

(c) $20^{\circ}$

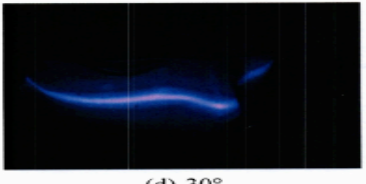

(d) $30^{\circ}$

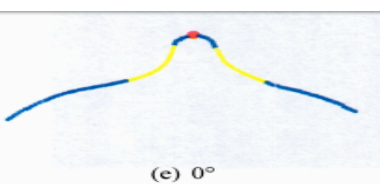

(e) $0^{\circ}$

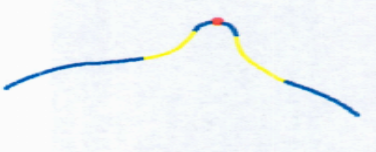

(f) $10^{\circ}$

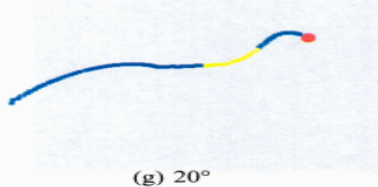

(g) $20^{\circ}$

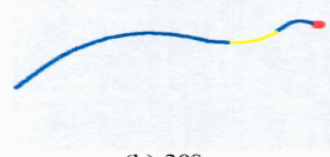

(h) $30^{\circ}$
Fig. (7). Experiments image.

\subsection{Analysis of Experimental Error}

In this experiment, the infrared camera is the low sensitive camera, and the stripes used for the projection are quite wide. Therefore, the contrast between the stripes and the surrounding background is a little lower than that under the visible light emission. More impurities appear among the extracted stripes. Some useful information is inevitably discarded accompanying the kicking out of the impurities, which will result in the incompletion of the stripe extraction. This is the real reason for the increase of the experimental errors.

Based on the analysis of the experimental results, it's found that the rotation angle can be accurately detected within a rotation range of $\pm 50^{\circ}$. The average error for the rotation angle is about $1.3^{\circ}$ or so, and the maximum error is 
about $3.0^{\circ}$. The nose detection method and the integrated calculation method are applicable within this range. As for the rotation angle within $\pm 60^{\circ}$ and $90^{\circ}$, the experimental errors seem quite large with an average error of $10.6^{\circ}$ and the maximum error of $19^{\circ}$. Since the focus of the projection light may not be properly adjusted, the light projected on the nose apex becomes blurred in the case of large rotation angle, and the stripe extraction becomes incomplete. This would result in an inaccurate position for the nose apex extracted from the stripes, and thus introduces a larger error to the experimental results.

It can also be found that the experimental results deduced under the infrared light projection are better than those under the visible light projection at a rotation angle of 40 or 50 degrees. For example, Fig. (8a and $\mathbf{8 c}$ ) are the experimental images at a rotated angle of $+40^{\circ}$ (right turn) under the infrared light emission and the visible light emission, respectively, and Fig. (8b and 8d) are the extracted images on the $x-z$ plane from their corresponding three-dimensional data which are relative to Fig. (8a and $\mathbf{8 c}$ ) respectively. Comparing Fig. $(\mathbf{8 b})$ with $(\mathbf{8 d})$, it could be seen that the extracted stripes are not identical even if same rotation angle are adopted. The positions of the stripes extracted under infrared light projection are slightly higher than the stripes under the visible light projection, and thus the stripes in Fig. (8b) seem a little moderate than those in Fig. (8d). It's interesting to found that the stripes projected on the nose in Fig. (8b) are not totally checked out, but the deduced position of the nose apex marked as red dot is correctly coincident with the real nose apex point. The adopted criterion that the fringed point is judged as nose apex is proved to be a powerful method. On the contrary, the stripes projected on the nose in Fig. (8d) is perfectly picked up, but unfortunately the deduced nose apex marked as black dot is not correctly coincident with the real nose apex point because of the imperfect nose apex judgment method.
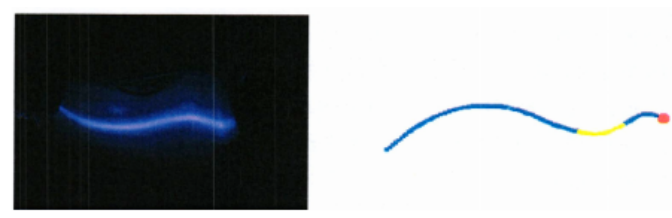

$\begin{array}{lll}\text { (a) Turn right at } 40 \text { degrees image (infrared light) } & \text { (b) } 40 \text { degrees of three-dimensional data in }\end{array}$ the $x-z$ plane (infrared light)
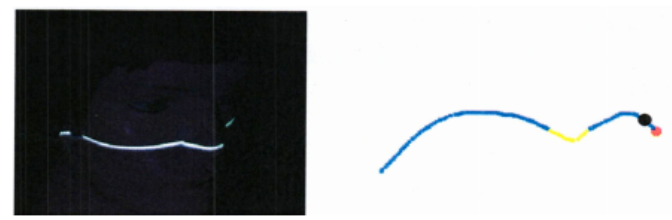

$\begin{array}{lll}\text { (c) Turn right at } 40 \text { degrees image (visible light) } & \text { (d) } 40 \text { degrees of three-dimensional data in }\end{array}$ the $x$-z plane (visible light)

Fig. (8). Experimental error analysis chart.

\section{CONCLUSION}

The current methods for the measurement of the rotation angle of the driver's face are briefly introduced in this paper, and an effective method based on three-dimensional image data measurement is proposed. The measurement of the rotation angle of the face needs to be considered such as ambient light, glasses, masks and other conditions. In this paper, a system for measuring the rotation angle of face based on visible light fringe projection is proposed. In the range of $\pm 90^{\circ}$ of the rotation angle, the average error of the experimental result is 2.3 degrees, the maximum error is 6 degrees, which meet the measurement requirements within \pm 90 . In order not to give the driver a burden, and to avoid the impact of ambient light, a measurement system based on infrared light projection is also constructed. The experimental results show that the average error of 1.3 degrees and the maximum error of 3 degrees are deduced within a rotation range of $\pm 50^{\circ}$, which can also satisfy the experimental requirement in the range of $\pm 50^{\circ}$.

Under the condition of infrared fringe projection, the measurement error increases with the increase of angle when the rotation angle is greater than 60 degrees. This is because the depth of focus for the projection light is too small, which results in the inconsistent clarity for the stripes taken by the camera. The stripes corresponding to the parts away from the camera are blurred out, and extracted stripes from the image data are incomplete. Therefore, it is necessary to do further research on how to enlarge the range of the projection light in the depth direction. Through the experimental results of visible light stripe projection, we can also find that the vertex of the nose can be detected correctly or is one of the reasons for the formation of errors. In the future, the further research will be done on the precise detection of the vertex of the nose.

\section{CONFLICT OF INTEREST}

The authors confirm that this article content has no conflict of interest.

\section{ACKNOWLEDGEMENTS}

Declared none.

\section{REFERENCES}

[1] W. Li, "Face recognition algorithm implemented on smart phones," Computer Technology and Development, vol. 1, pp. 161-164, 2008.

[2] Li Yang, "Safe driving in the driver's face to the detection of research", Scientific and Technical Information, vol. 38, pp. 29-31, 2009.

[3] D. Dssilva, and D. Metaxas, "Optical flow constraints on deformable models with applications to face tracking", International Journal of Computer Vision, vol. 38, pp. 99-127, 2000.

[4] R. De, "The new focus of US highway traffic safety", Integrated Transport, vol. 1, pp. 75-77, 2009.

[5] D. Chai, and A. Bouzerdoum, "A Bayesian approach to skin color classification in $\mathrm{YCbCr}$ color space”, Conference Proceedings on TENCON, vol. 2, pp. 421-424, Kuala Lumpur, 2000.

[6] R. Wang, and K. Guo, "A monitoring method of driver fatigue behavior based on machine vision", Conference Proceedings on IEEE Intelligent Vehicles Symposium, USA, 2003.

[7] J. Li-sheng, and T. Lei, “An Improved Otsu Image Segmentation Algorithm for Path Mark Detection under Variable Illumination", USA, 2005, pp. 657-662.

[8] K. Shearer, and K.D. Wong, "Combining multiple tracking algorithms for improved general performance", Pattern Recognition, vol. 34 , pp. 1257-1269, 2001. 
[9] S.K.L. Lal, and A. Craig, "Development of an algorithm for an EEG-based driver fatigue countermeasure", Journal of Safety Research, vol. 34, pp. 321-328, 2003.
[10] M. Bertozzi, and A. Broggi, "Vision-based intelligent vehicles State of the art and perspectives," Robotics and Autonomous Systems, vol. 10, pp. 1-16, 2000

(C) Dongmei and Shengmin; Licensee Bentham Open.

This is an open access article licensed under the terms of the Creative Commons Attribution Non-Commercial License (http://creativecommons.org/licenses/by-nc/4.0/) which permits unrestricted, non-commercial use, distribution and reproduction in any medium, provided the work is properly cited. 